\title{
Potenciamiento de la Autonomía en los Miembros de la Familia: Una Proposicion para Asumir el Riesgo en la SOCIEdAd CONTEMPORÁNea
}

\author{
Por: Liliana Pérez Mendoza
}

\section{RESUMEN}

\begin{abstract}
7 l artículo presenta un análisis de la familia en la sociedad actual, que ha sido llamada por autores como Beck, "sociedad del riesgo", mostrando como ésta última, en la medida en que exige mayor individualización de los miembros de la familia, también demanda de relaciones más democráticas a su interior, lo cual abre paso para propuestas de intervención social, que potencien la autonomía de sus miembros, desde una constelación conceptual que señale una perspectiva de la autonomía de tipo solidaria, reflexiva, comprensiva y dialógica, como la que se deriva de la teoría de la acción comunicativa de Jürgen Habermas.
\end{abstract}

\section{El Riesgo, un Planteamiento Inicial}

Para la sociedad contemporánea, la idea de estar en riesgo permanente, toma cada vez mayor sentido, por cuanto éste ha sido considerado intrínseco al pensamiento y al actuar de las personas. Uno de los autores que ha plantado tal teoría es Ulrich Beck, quien señala una sociedad donde el riesgo está presente como aquella «(...) fase de desarrollo de la sociedad moderna en la que los riesgos sociales, políticos, económicos e individuales tienden cada vez más a escapar a las instituciones de control y protección de la sociedad industrial». (Beck, Giddens y Lask, 1997: 18).

Por ello consideran, que la sociedad actual se mueve entre factores que amenazan su estabilidad y la búsqueda de seguridad, de tal forma que la conciencia colectiva ha incorporado la inseguridad incluso en el plano de la familia, como algo propio en su cotidianidad. Por lo cual, pareciera que la familia entra en una fase de desencantamiento, y el peso de las decisiones recae entonces en los individuos, lo que produce un proceso de individualización a su interior, donde cada persona 
pasa a elaborar su biografía y se generan nuevas formas de vida, de manera no casual pero si involuntaria.

Por tanto, fenómenos como la individualización y la solidaridad, que acompañan el proceso de modernidad/modernización, se constituyen por así decirlo, en «cara y sello» de una misma moneda, que en el caso de la familia, conllevan a que ésta sea intervenida a partir de la reflexividad, el diálogo, el consenso racional, a fin de que la conquista progresiva de autonomía de cada uno de sus miembros sea una realidad, en la medida en que éstos reconozcan que la misma es un derecho de todos, y por lo tanto, actúe con respeto hacia todo "otro", de forma solidaria, comprensiva y responsable.

Lo cual no implica que las identidades y competencias de cada uno de sus miembros se desconozcan, tergiversándose los roles de padres e hijos, así como los de los demás miembros de este sistema, produciéndose un desencanto en las expectativas y sentidos que tiene para cada uno la familia, sino que por el contrario, éstas sean consideradas para un ejercicio dialógico permanente y fecundo, por cuanto señalan modos de "ver" y "voces" diferentes a considerar en el establecimiento y aceptación de normas por parte de cada uno, para guiar de forma responsable sus practicas a su interior y en la relación al sistema con la sociedad.

Es por ello que de lo que se trata en la familia, tal como en la educación, es de preparar a los sujetos para esta sociedad contemporánea, que se enmarca en lo que Beck denomina, una modernización reflexiva, referida al proceso de pasar de la sociedad industrial a la sociedad del riesgo, ${ }^{1}$ y donde las personas se adentran en esta sociedad global del riesgo, como individuos libres e iguales para el dialogo reflexivo, participativo y deliberativo, pero también como personas necesitadas y habilitadas para actuar de manera solidaria con los "otros".

\section{La Familia: Entre la Individualización y la Democratización}

1 “(...) modernización reflexiva significa autoconfrontación con aquellos efectos de la sociedad del riesgo que no pueden ser tratados y asimilados dentro del sistema de la sociedad industrial, en tanto, que medidos por los e $\mathrm{s}$ t á $\mathrm{n}$ d a $\mathrm{r}$ e $\mathrm{s}$ institucionalizados de esta última (Beck, Giddens y Lask, 1997: 18-19).
Beck $(1997,1998)$ destaca el proceso de individualización, y lo distingue drásticamente de la atomización, aislamiento, soledad o desconexión, incluso de la emancipación o resurgimiento del individuo burgués, situándolo como un proceso hacia nuevas formas de vida, no por casualidad ni voluntariamente, sino de una vez y en las condiciones generales del Estado de bienestar, en la sociedad industrial avanzada. Así pues, estamos en medio de un proceso imparable de mundialización, condenado a la individualización como una compulsión que sirve para elegir una biografía entre diferentes modelos. Todo 
ello, en una sociedad nueva que sustituye a otra sin revolución: La sociedad del riesgo, que es el resultado de la modernización, de un capitalismo que lleva a la agudización e individualización de las desigualdades sociales, haciendo que las crisis sociales, las familiares, parezcan crisis personales.

Este proceso de individualización, que abarca tanto a hombres como a mujeres, sin restricciones de clase social, hace que se vuelvan cuestionables los pensamientos e investigaciones que, como en el caso de la familia, partan de categorías o estamentos como clases o capas. Así, mientras las personas aún se comunican dentro de las mismas instituciones y maneras, como la familia; también se retiran en busca de nuevas actividades e identidades, en un tipo de práctica que ni es instrumentalmente racional ni está determinada por roles, pero donde la opinión pública, los movimientos sociales, los grupos de expertos y la gente trabajadora tienen un papel básico: Configurar la sociedad desde abajo, donde los individuos son los protagonistas.

Según Guy Bajoit, «Estamos viviendo, desde hace tres o cuatro décadas, en una época de mutación cultural: estamos pasando, poco a poco, de un modelo industrial (en el cual el individuo, para dar sentido a su existencia, tiene que someterse a las exigencias de lo colectivo: hacer su deber, someterse a la disciplina de las instituciones) a otro modelo, que lo llamaremos identitario (en el cual el individuo afirma su autonomía, su derecho a la autorrealización personal, la prioridad de las exigencias de la persona)», donde hay un llamado al individualismo que se traduce en cuatro mandamientos esenciales a saber: «es un llamado a la autorrealización de si mismo: "sé tu mismo», o más bien, «vuélvete tu mismo, en el curso de tu vida»; «busca en ti lo que eres, descúbrelo por experiencias, y realízalo»;- es, por ende, un llamado al libre arbitrio: «sé autónomo, sujeto de tu vida, toma tus opciones, decide para ti, no te dejas imponer nada por los demás (salvo si te conviene)»;- es también un llamado al placer: «goza de la vida, aquí y ahora, vive con pasión, con emoción» (y por supuesto: «no sufras»!); y, finalmente, es un llamado a la prudencia: «cuídate: tu vives en un mundo de riesgos» (2004: 3). Es decir, estamos hablando de un ideario de individualismo puro que para nada entiende de solidaridad, salvo si con ella obtiene más que beneficios personales.

De cualquier manera, Beck no examina la sociedad del riesgo como algo problemático que nos ha de llevar a un callejón sin salida porque: "la sociedad de riesgo puede poner en marcha también un proceso de aprendizaje, una politización forzosa, posibilidad perfectamente imaginable como proceso de aprendizaje mundial, como cosmopolitización...»(Beck, 2002: 95-216). 
Y esto es correspondiente en la intervención social con familias con el enfoque de resiliencia, la cual es definida como la capacidad que tienen los sujetos para sobreponerse a la adversidad y que «surge de la inquietud por identificar aquellos factores que permiten a las personas sortear las dificultades y condiciones adversas que se le presentan en su vida cotidiana de manera exitosa. Estos factores de origen social o individual según las investigaciones recientes pueden modificar el concepto tradicional de factores de riesgo, pues una situación adversa en lugar de tener una consecuencia fatal, puede constituirse en un factor positivo o de resiliencia que contribuye al mejoramiento de las condiciones de vida de una persona y su entorno» (Badilla, 1999: 22), lo que para algunos autores es «inverso al riesgo o complementario a él». (Ibíd., 1999: 22).

Por lo tanto, «la resiliencia no es una característica o dimensión estática. Es la articulación continua de capacidades y conocimiento derivados a través de la interacción de riesgos y protecciones en el mundo» (Saleebey, D., 1996. Citado por Badilla, 1999: 24), por ello, otro aspecto que considera la teoría de la sociedad del riesgo, es que ésta «se vincula estrechamente a procesos complementarios de individualización en los ámbitos del trabajo remunerado, de la familia, de las relaciones de género, de la biografía reflexiva y de la autoidentidad» (Beck, op. cit.: 18). Es por ello, que «la investigación y abordaje de los factores riesgo asume un nuevo rostro cuando se analiza bajo el prisma de la resiliencia» (Badilla, op. cit.: 25).

Es aquí, cuando el abordaje de la autonomía de cada uno de los miembros de la familia, se constituye en una de las principales competencias a fortalecer a través del dialogo, de la comunicación, porque es la familia la instancia inicial, donde tienen lugar los primeros ejercicios participativos y deliberativos de las personas, formándolas en la reflexividad, entendiendo ésta como la «autoconfrontación, autotransformación» (Beck, op. cit.: 28), a través del dialogo, lo cual a su vez, ha de ser validado, reproducido y cualificado posteriormente en otros ámbitos de la sociedad como son: el educativo, el laboral y el comunitario.

De forma tal que desde la familia, se fomente la actuación de cada uno de sus miembros, más ética en tanto que solidaria, dirigida hacia un entendimiento reciproco sobre normas de actuación que guíen aceptada y responsablemente las practicas sociales de cada individuo en la familia y en el mundo social, como mecanismo para asumir el riesgo que se suscita permanentemente en esta sociedad, donde tiene lugar «la metamorfosis del peligro, difícil de delimitar y de controlar» (Ibíd.: 21). 
También hemos de reconocer que en la actualidad, las sociedades son cada vez más dialógicas (Flecha y otros, 2001), ya que el diálogo está penetrando cada vez más en todos los ámbitos, tanto públicos como privados, políticos y personales. De esta manera, se abren nuevas formas de convivencia más igualitarias e inclusivas. En este contexto es posible convivir, dialogar y reflexionar conjuntamente diferentes grupos culturales, personas con diversas opciones sexuales o familiares, hombres y mujeres, niñas y niños, personas adultas, etc.

Si tenemos en cuenta que la sociedad moderna, tiene como proyecto la autonomía y la ciudadanía del hombre, entonces la familia, debe posibilitar que cada uno de sus miembros contribuya a resolver a su interior sus conflictos, respetando las diferencias de pensamiento y de competencias identitarias de sus participantes, reconociendo que es mediante el dialogo libre e igualitario, como es posible llegar a acuerdos sobre la actuación consensuada y responsable, porque la autonomía es posible cuando se reconoce el mismo derecho en los demás y se actúa en consecuencia con respeto hacia cada uno, pese a lo diverso que pueda ser, pues de esta manera cada uno también es prójimo. Es por ello que en la sociedad actual, el desafío está en la consideración del dialogo y de sus propósitos, lo cual también debe estar haciendo parte de la agenda de las intervenciones sociales con familias, pues de lo que se trata en últimas es de una perspectiva de intervención de segunda generación, más compleja en tanto, permita el reconocimiento de los derechos de las personas e intente las diferencias.

Ahora bien, uno de los grandes cambios en las últimas décadas del siglo XX y lo que llevamos del siglo XXI, es la transformación de las estructuras familiares y las formas de relación. Las familias están cambiando, como ya venía anunciando el aumento de divorcios, de parejas no casadas que conviven, de hogares monoparentales y homoparentales, etc., y son estas características las que señalan que estamos también ante una familia en crisis. En Chile, por ejemplo, de acuerdo con los datos proporcionados por CNF (1993), entre el 9.2\% y el 14\% de las familias son recompuestas, mientras que según el Censo del año 2002, el porcentaje de familias monoparentales es de 9.7\% (Gubbins, et. al, 2003). Igualmente, el Censo del mismo año señala que el $9 \%$ de

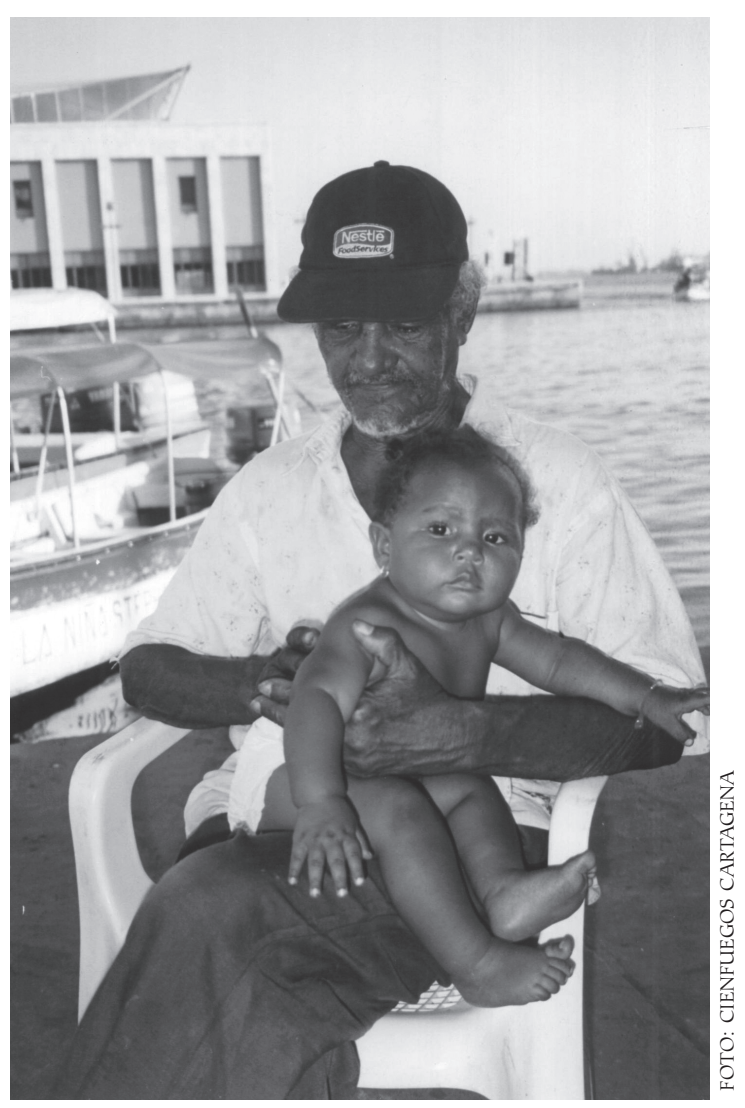


los chilenos conviven con su pareja, mientras que el número de matrimonios se reduce del año 2000 al año 2003, pasando de 62.073 a 57.682, y las nulidades aumentan de 6.654 en el año 2000 a 7.080 en el año 2003 (Datos de Registro Civil).

Es por ello, que otros autores como Giddens (1995), nos explican que estamos también ante una democratización de la vida personal. La democracia, ha penetrado en la vida más intima y, de esta manera, las formas familiares patriarcales y jerárquicas se van destruyendo. No se trata de una pérdida de valores ni de crisis familiar, sino de la incorporación de valores más democráticos en las relaciones que en el proceso de transformación hacia relaciones más igualitarias van dejando en el camino las formas de relación hegemónicas. Tal "democratización en el contexto de la familia implica igualdad, respeto mutuo, autonomía, toma de decisiones mediante la comunicación y la ausencia de violencia» (Giddens, 1999: 112).

Es claro entonces, el sentido que tendría potenciar en la familia, el componente de la autonomía, de la igualdad y del respeto mutuo de cada sus miembros a través del dialogo, como forma no violenta de resolver los conflictos, y esto podría ser posible a través de una intervención social familiar para la promoción del empoderamiento de cada uno de sus miembros, lo que significaría creer que cada persona es capaz de tomar sus propias elecciones y decisiones, porque el ser humano no solo posee la fuerza y el potencial para resolver sus propias situaciones difíciles, sino que también es capaz de aumentar su fuerza y así contribuir a la sociedad (Aylwin y Solar, 2002), pero significa reconocer que es en la integración social, en la socialización con el otro al interior de la familia, donde cada uno ejerce su libertad, y su identidad y eso significa actuar en una perspectiva de comprensión y solidaridad con cada "otro", porque todos y cada uno tienen los mismos derechos y por lo tanto, está habilitado para participar independiente y responsablemente.

Esto contrasta con lo que Beck señala acerca de la relación inversa entre la democratización de la vida personal y la democratización de la familia, pues para él aún no puede hablarse precisamente de una democratización de la familia, pues aunque «es posible que las viejas estructuras de autoridad estén deterioradas; su barniz en todo caso ha desaparecido» (1999: 209), ya que para el mismo autor, «los principios del diálogo del cambio eventual de roles, del escuchar, del hacerse responsable por el otro siguen incumplidos" (Ibíd.: 209), sin embargo, no se trata de resignarse a ello, sino precisamente, de esforzarnos cada vez más por hacer frente a este "riesgo" en la sociedad, y procurar 
intervenciones sociales, políticas sociales, que potencien la autonomía y la democratización como derechos, y como anhelos posibles de alcanzar en una modernidad que aun no esta concluida.

Si tenemos en cuenta que según Elizabeth Beck-Geirnsheim, «la familia no se está resquebrajando...»(2003: 66), sino que «está adquiriendo una nueva forma histórica», que ella denomina la emergencia de una «familia posfamiliar» (Ibíd.: 166), la cual con el proceso de individualización hace que ésta pierda su función de unidad laboral y económica e inicie una nueva relación con el mercado laboral (Ibíd.: 171), aflojándose considerablemente los lazos familiares, dada la nueva lógica de las biografías individuales, es aquí cuando hemos de considerar, que no tiene sentido contribuir a la construcción individual cuando los lazos familiares y sociales se rompen a costa de esta "biografizaciòn del individuo", pues en esta carrera lo que se fomenta a largo plazo es un individuo que actúa de manera instrumental hacia su familia y la sociedad, en tanto, pretende utilizar su pertenencia e dichos ámbitos para fines individuales, dejando atrás la posibilidad de una actuación hacia un entendimiento racional y comunicativo con los otros para resolver pacíficamente las diferencias, porque los demás, son también sujetos de derechos.

\section{Algunos Cambios Familiares y Sociales que lo Explican...}

Y es que el dialogo en condiciones de libertad, igualdad y solidaridad, es la opción para la reconstrucción de la familia, y de otros ámbitos de la sociedad, ya que por ejemplo, en el caso de la familia, sin lugar a dudas, la emancipación femenina ha sido decisiva en los cambios de las relaciones a su interior, pues el rechazo a los roles tradicionales de la mujer como madre y esposa, y su acceso al mercado laboral y a la educación han propiciado un cambio radical y continuo en éstas. En efecto, la pérdida de las identidades tradicionales, en el caso de la mujer, como ha sido descrito por Beck y Beck-Gernsheim (2001), está generando, que todo aquello que se daba "por descontado» en las relaciones, ahora pueda ser y tenga que ser decidido cada vez más libremente por las personas.

La familia, la pareja, el amor, la sexualidad ó la relación con las hijas e hijos ya no pueden presuponerse. Siendo ajenos al amparo de la tradición, la decisión es más libre pero también genera una mayor inseguridad y riesgo. El resultado de esta situación de nuevo no conlleva una pérdida de valores familiares, sino la convivencia de una cada vez más extensa pluralidad de opciones de vida, familiares y sexuales. Además, colectivos tradicionalmente excluidos como los formados por gay's, lesbianas, transexuales, etc., reclaman su espacio, así como 
ser escuchados e incluso reconocidos como familia.

Por lo cual, una intervención social con familias que inserte la perspectiva de los derechos, es una invitación a recorrer un camino que desde la comunicación, reconozca y respete sus diversos miembros, en tanto, cada uno tiene la posibilidad de erigir a través de sus discursos sus diversas pretensiones de validez para construir normas practicas consecuentes con estas nuevas realidades, donde ya las normas anteriormente establecidas por cada sociedad, ya sencillamente, no aplican, por cuanto han dejado de generar entre sus miembros un actuar reflexivo, responsable y consecuente con las mismas, constituyéndose por tanto, en normas vacías de sentido y de acción, y donde el actuar libre de las personas en la familia y la sociedad, no ha dado aún para reconocer la legitimidad de los discursos de cada uno, en un escenario de entendimiento en la construcción consensual de normas practicas por parte de todo "otro" participante de la dinámica familiar, lo cual tiene mayor asidero si consideramos que en la contemporaneidad, las familias ya no se reducen a una estructura de familia nuclear organizada de forma jerárquica, sino más democrática.

Lo cual no implica desconocer que no han desaparecido las familias nucleares, en las cuales, además de que su forma no implica ya una estructura jerárquica ahora conviven con las familias monoparentales, homosexuales, heterosexuales con hijos e hijas de diferentes parejas anteriores o con parejas que viven separadas, etc., pues de hecho, con la destradicionalización de los roles de género y las relaciones, y en la búsqueda de combinar éstas, se establece el desarrollo de cada biografía individual donde se van creando estas nuevas formas familiares que requieren del diálogo continuo para su formación y mantenimiento. Por lo que no se trata que la socialización, vinculada en primera instancia en la modernidad tradicional al espacio familiar, deje de ser considerada como tal y se entienda como una autosocialización, donde la vida propia «se ha colocado en el lugar de la autoridad paternal, maternal o de los gobernantes, maestros policías ó políticos» como señala Beck (1999: 205), sino que tal "vida propia", reconozca las diferencias identitarias y de competencias de cada uno de los miembros de la familia, y más tarde de la sociedad, y las considere como la posibilidad para generar un dialogo más fructífero, en tanto reconoce y posibilita la expresión de la diversidad, esa diversidad que es también prójima, permitiendo con ello, comprender miradas distintas de una misma actuación, en el establecimiento conjunto, aceptado y espontáneo, de formas de acción universalizables para las prácticas responsables y comprometidas de cada uno de sus miembros. 


\section{La Intervención Social en la Familia Contemporánea}

En la contemporaneidad, cada vez más, la familia requiere posibilitar a su interior una mayor participación reflexiva y discursiva de cada uno de sus miembros a fin de articular a partir de lo que algunos llaman un ejercicio de equilibrio cotidiano, las diferentes biografías individuales que se entrecruzan en ella, y que buscan un camino diferenciado, a través de la búsqueda de equidad en la distribución de los recursos y la apertura hacia nuevos espacios y ritmos de socialización elegidos por sus miembros. Ya no es posible seguir pensando que cada miembro de la familia tenga el mismo concepto de ella, pues cada uno tiene su propia concepción sobre la misma y lo que ella implica para si mismo, siendo además, el vinculo familiar una elección que cada individuo asume libremente, y esta es otra característica de individualismo en esta nueva etapa de modernidad, pues ahora «cada individuo traza sus propias fronteras» (E. Beck-Gernsheim, op. cit.: 184), haciendo que los vínculos sean más finos, frágiles y flexibles, pero también, que dicha individualidad dependa de la solidaridad y de los riesgos internos y externos.

Por otro lado, la capacidad de una familia para resolver estos problemas parece que guardara una relación inversamente proporcional entre el potencial interno de conflictos que se generan y la distribución de la autoridad y el poder de algunos de su miembros. Por cuanto entre más desigualmente está distribuido y asumido el poder entre los miembros (adultos) de la familia, mayor es la probabilidad de una distribución asimétrica de las oportunidades que cada uno tiene de establecer una gratificación satisfactoria. Pero los conflictos resultantes de esto, no pueden manejarse en términos abiertamente estratégicos en el marco de la familia, porque están directamente implicados con la búsqueda de la identidad de los participantes. Una familia con constelaciones de poder, que no permiten resolver el problema de una equilibrada satisfacción de las necesidades entre sus miembros, estrecha el espacio para una adecuada gestión de la propia identidad de cada uno.

Por estas razones, la familia pasa a volverse para cada miembro «más una relación electiva, una asociación de personas individuales, cada una de las cuales aporta sus propios intereses, experiencias y planes, y está sometida a diferentes controles, riesgos y condicionamientos»(Ibíd.: 185), la generación de autonomía toma un carácter de mayor despliegue para cada individuo en solitario, por lo que ésta bien podría ser una de las tareas a asumir en la intervención social, pues pese al desarrollo progresivo de estos procesos de individualización, la familia sigue siendo una instancia consultiva y en ese sentido, de cierta dependencia de sus miembros, aunque ya no decisoria de las elecciones individuales, y es además, el primer espacio donde se reconocen y estrechan los lazos sociales y de las 
personas, a través del dialogo libre, aunque la mayoría de las veces, no igualitario.

Tales transformaciones en la familia es claro que tienen repercusiones en muchos ámbitos, sobre todo en el educativo, ya que los niños y jóvenes que se forman en los establecimientos educativos pueden pertenecer a formas familiares muy diversas y también cada vez más democráticas, por lo cual, los textos o libros, las asociaciones y reuniones de padres, las preguntas en el aula, los valores, y las competencias a desarrollar, deben ser revisadas y trasformadas a partir de la reflexión sobre la nueva realidad familiar. Por lo que, el espacio educativo, no puede seguir transmitiendo ni reforzando un único modelo familiar tradicional, pero tampoco puede rechazarlo, sino que debe incluir la pluralidad de opciones sexuales y familiares para no excluir a ningún niño o joven, y para fomentar el diálogo y la reflexión conjunta sobre la pluralidad de opciones de relación familiar así como la elección personal de familia, con base en su propia historia, en el desarrollo de su autonomía, pero también en la comprensión y solidaridad con el "otro" con quien comparte un lugar llamado "familia".

También hoy en día, ante la situación actual de la extensa violencia de género, es necesario cuestionarse el modelo de relación amorosa, de pareja, etc., vivenciado y asumido por cada miembro en el seno familiar. Los malos tratos son la expresión más explícita del deterioro de una relación, y la ayuda en la superación de los modelos irracionales de comportamiento amoroso es uno de los cambios más radicales e importantes que se pueden promover desde una intervención social más reflexiva y dialógica, donde el componente de la generación de autonomía del tipo solidaria, en los miembros de la familia, es importante, ya que los valores, las normas y conflictos que se den en éste ámbito familiar, deben

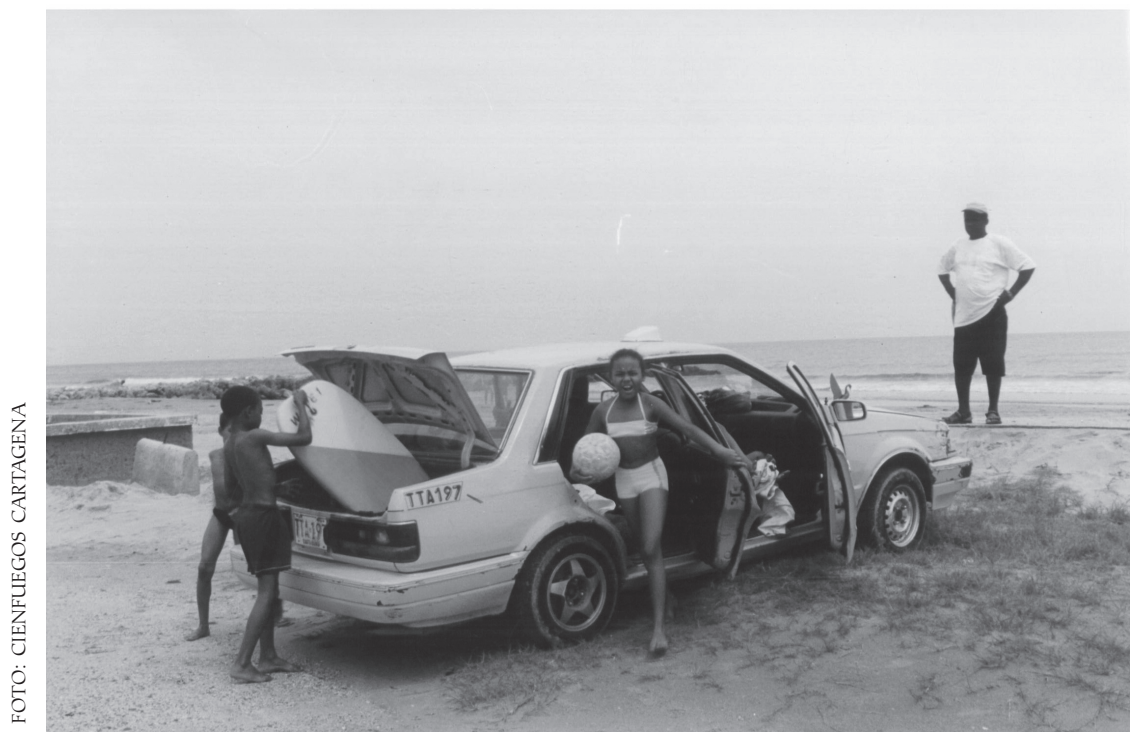
ser reflexivizados por todos $y$ cada uno de los miembros a través del diálogo democrático en el que se respeten la voz y los derechos de todas las personas, sobre todo los de libertad, igualdad y solidaridad, frente a las expectativas, intereses, planes y elecciones de cada uno de sus miembros, independiente de sus identidades tradicionales y competencias familiares.

Aquí es importante considerar 
otro elemento, que tiene que ver con las comunicaciones distorsionadas, que siguen a problemas de forjamiento de la identidad del yo, las cuales no sólo se presentan en el ámbito de las relaciones familiares, pero sí que lo hacen con particular frecuencia, por ello se trata de reconocer que la familia se constituye en un lugar de privilegio para la gestión de la propia identidad, no sólo para los niños, que en ella empiezan construyendo su identidad, sino también para sus miembros adultos, que la resignifican, en tanto, de lo que se trata desde esta perspectiva de la autonomía solidaria, comprensiva y responsable, es de que cada mimbro de la familia se sienta comprometido, por que así lo entiende y lo desea, a actuar hacia un entendimiento comunicativo y en consecuencia, no actúa pues perdiendo lo que considera hace parte de su identidad y competencias, en este caso familiares, es decir, como padre, madre, hijos/as, esposo/a, abuelos, abuelas, tías/os, sobrinos/as.

\section{Una Intervención Social Familiar desde la Autonomía Habermasiana}

La familia entonces, deja de ser por excelencia reguladora de relaciones interpersonales y continua siendo un ámbito íntimo de relaciones cara a cara que se enmarca en una estructura de roles y autoridad, ahora mediados por la comunicación libre, igual y solidaria entre sus miembros, hacia el entendimiento sobre el establecimiento de normas practicas que guíen el actuar de cada uno de sus miembros ante sus nuevas situaciones y realidades, y que a su vez se reconstruyan permanentemente cuando ya dejen de ser validas para la actuación de al menos uno de sus miembros.

Por ello, el concepto de autonomía trabajado por Habermas, se constituye en un planteamiento interesante para abordar la familia contemporánea, por cuanto para este autor "la autonomía conlleva una idea de solidaridad comprensiva, ya que es ella y sus movimientos de conmoción, las que informan acerca del mejor modo de comportarse para contrarrestar mediante la consideración y el respeto la extrema vulnerabilidad de las personas. Esta vulnerabilidad es aquella que está inscrita en las formas de vida socio-culturales, ya que la individuación se produce a través de la introducción en un mundo de la vida intersubjetivamente compartido" (Habermas, 1991: 106).

Es decir, en el ámbito familiar, la autonomía sería algo más que la capacidad de cada individuo para tomar decisiones, sino que es, por así decirlo, la forma de contribuir a que cada individuo o miembro de la familia desarrolle su propia reflexividad, mediante el diálogo y el entendimiento con los otros. Y estos, son los fines que una intervención social con familia para la autonomía de cada uno de sus miembros, debe perseguir. En otras palabras, podríamos decir que en 
una intervención social con familia - desde la perspectiva habermasiana - la autonomía significa reflexividad, discursividad, y consenso en la medida en que cada miembro de la familia es asumido por todos como participante de una acción racional comunicativa, mediante la cual toma conciencia de sí mismo, de su familia y del mundo social del que hace parte.

Este tipo de intervención también es válida, si se considera que desde el punto de vista de las funciones que el sistema familiar tradicionalmente ha cumplido para sus distintos miembros, se presentan varios problemas: el de una satisfacción plena de las necesidades, el de un forjamiento de la identidad, el que resulta del punto de vista complementario de qué es lo que han de aportar las interacciones de los miembros a la estabilidad del sistema familiar, el aseguramiento de la autonomía del sistema y la autorregulación del intercambio de la familia con su contexto y finalmente, el que define la relación funcional de la familia con el sistema social global. Por lo que, uno de los dispositivos a generar en cada miembro de la familia, es su capacidad de reflexivizar su contexto familiar y en esa medida contribuir al fomento de su autonomía y el reconocimiento y respeto por todo "otro" como legitimo miembro de la familia y por tanto con igual capacidad racional y discursiva, para afrontar de manera aceptada y co-responsable estos y otros problemas que puedan surgir eventualmente en este sistema.

Volviendo al tema de los riesgos, éstos «no se agotan en circunstancias ó daños que ya han tenido lugar, sino que contienen esencialmente un componente futuro. Este reposa tanto en la prolongación al futuro de los daños ya visibles como en una pérdida general de confianza o en la suposición de un «fortalecimiento del riesgo» (Beck, 1998: 39), por lo que aquí el prepararse para los riesgos conocidos tan sólo es la base para considerar que siempre habrán riesgos menores o mayores, en la misma o en otra dirección, pero cada vez más creciente en su dinamismo interno; por ello la solidaridad pasa a ser un principio de las relaciones sociales y familiares, pues la sociedad del riesgo implica también protección ante el miedo que éste genera en la sociedad, aunque para algunos autores «esta obligación de solidaridad también está perdiendo consistencia» (Beck-Beck-Gernsheim, op. cit: 174).

Ahora bien, la participación de las familias en los procesos educativos sigue siendo esencial, ya que el aprendizaje depende también de la correlación de lo que se aprende dentro y fuera de este ámbito social, por lo que resulta importante la acción conjunta entre la familia y los diferentes ámbitos de la sociedad donde sus individuos interactúan. Por lo que en este punto, cabe preguntarse más acerca 
de los temas que deberían trabajarse en una intervención social con familias para la autonomía como competencia comunicativa de cada uno de sus miembros. Es por ello que se debe partir entonces, que la familia debe educar a sus miembros a través de valores positivos que contribuyan al establecimiento de relaciones satisfactorias por parte de cada uno, y para ello, es necesario crear contextos formativos que faciliten estos procesos pero no controlarlos.

Se trata entonces, de potenciar intervenciones sociales con familias, que contribuyan a formar en el reconocimiento y respeto por cada uno de sus miembros, como un sujeto de derechos, con capacidad de habla y de acción para llegar a un consenso racional, que permita relaciones más democráticas, que resignifiquen las identidades y competencias tradicionales, pero también conocedora de los riesgos que esto comporta tanto para la persona, como para la misma familia y la sociedad en general. Todo ello dependerá del grado en que se logre que la familia se cierre o se abra, de la medida en que se constituya como una unidad a través sus miembros, en donde las redes internas de comunicación queden ligadas a instancias situadas dentro y fuera del sistema familiar, habilitándose como un espacio real para la resiliencia, no evitando o desconociendo las experiencias de riesgo a que pueda estar sometida, sino más bien hacia la generación de factores protectores, que en conjunto y con la participación libre, igualitaria y solidaria de cada uno de sus participantes, contribuya desde el discurso reflexivo dirigido hacia el entendimiento mutuo sobre normas de actuación, a mitigar los impactos de esos riesgos para ella misma como unidad de la sociedad.

\section{BIBLIOGRAFIA}

\footnotetext{
AYLWIN, Nidia y Maria Olga Solar. (2002) Trabajo social familiar. Pontificia Universidad Católica de Chile, Santiago.

BADILLA, Helena. (1999) Para comprender el concepto de resiliencia. En: Revista costarricense de trabajo social. Costa Rica. Colegio de trabajadores sociales de Costa rica. Núm. 9. San José.

BAJOIT, Guy. (2004) A propósito de la eficacia de las políticas sociales del Estado. Bélgica. (Paper).

BECK, U.; Giddens, A.; Lash, S. (1997) Modernidad reflexiva. Alianza Editorial. Madrid.

BECK, U. (1998) La sociedad del riesgo. Hacia una nueva modernidad. Paidós. Barcelona.

BECK, U. (1999) Los hijos de la libertad. Fondo de cultura económica. Buenos Aires.

BECK, U. (2002) La teoría de la sociedad del riesgo reformulada. (Traducción de Fernando Robles). En: Gobernar los cambios: Chile más allá de la crisis. DOS. Ministerio Secretaría general de gobierno. Santiago.
} 
BECK, U.; Beck-Gernsheim, E. (2001) El normal caos del amor. Las nuevas formas de la relación amorosa. Paidós/El Roure. Barcelona.

BECK-Gernsheim, E. (2003). La reinvención de la familia. En busca de nuevas formas de convivencia. Paidós ediciones, Barcelona.

FLECHA, R.; Gómez, J.; Puigvert, L. (2001) Teoría sociológica contemporánea. Paidós. Barcelona.

GIDDENS, A. (1995) Modernidad e Identidad del yo. Península. Barcelona.

\section{BIOGRAFÍA}

\section{LILIANA PÉREZ MENDOZA}

Trabajadora social. Universidad de Cartagena. Especialista en Administración de Programas de Desarrollo Social y en Teorías, Métodos y Técnicas de investigación social. Universidad de Cartagena. Candidata Magíster en Trabajo Social, Pontificia Universidad Católica de Chile. Docente Facultad de Ciencias Sociales y Educación, Universidad de Cartagena. 